Thorax (1965), 20, 149.

\title{
Oxidative enzymes of the interalveolar septum of the rat $^{1}$
}

\author{
W. S. TYLER ${ }^{2}$ AND A. G. E. PEARSE \\ From the Department of Pathology, Postgraduate Medical School, London, W.12
}

Though the interalveolar septum is the target tissue for many deleterious agents, and one of its constituent cell-types is thought to be the source of a surfactant essential to normal pulmonary function (Klaus, Reiss, Tooley, Piel, and Clements, 1962), little is known concerning the metabolic potential of this tissue. Histochemical methods, though mainly qualitative, permit precise localization of chemical activities among various components of an organ. This type of information is not obtainable by the classical biochemical methods of extraction, fractionation or tissue incubation. The object of this study is to reveal the metabolic potential of the cells of the interalveolar septum.

The few histochemical studies that have been reported have concerned only a few of the histochemically demonstrable oxidative enzymes (Pliess and Suhr, 1959; Sorokin, Padykula, and Herman, 1959 ; Sorokin, 1961). The small number of studies appears to be due in part to the difficulty in preparing sections of lung suitable for dehydrogenase histochemistry. The authors have devised a simple method for preparing thin cryostat sections of distended lung which are suitable for histochemical procedures.

\section{MATERIALS AND METHODS}

Lungs from 10 female white rats weighing 150 to $250 \mathrm{~g}$. were studied. Six of the rats were euthanized by the inhalation of coal gas. The other four received Nembutal intraperitoneally. The thorax was opened and the lungs were allowed to collapse. The trachea was isolated in the cervical region, cannulated, and $4 \%$ gelatin (Bacteriological grade from G. T. Gurr) at $37^{\circ} \mathrm{C}$. was injected until the lungs approximately filled the thorax. The gelatin in the lungs was congealed by cooling in running cold water for five to 10 minutes. Small blocks of gelatin-infiltrated lung

1 Supported in part by U.S.P.H.S. Special Fellowship 1-F3-HE-20 317-01 and U.S.P.H.S. HE-06101

On Sabbatical leave from the Department of Anatomy, University of California, Davis, Calif., U.S.A. were quenched in liquid oxygen. Sections $8 \mu$ and $20 \mu$ thick were cut using a microtome cryostat maintained at $-15^{\circ} \mathrm{C}$., picked up on coverslips at room temperature, and allowed to thaw and dry at room temperature. All sections were incubated within three hours of being cut.

The histochemical methods were those given by Pearse (1960). The monotetrazolium-cobalt chelation method using 3(4,5-dimethylthiazolyl-2)-2,5-diphenyl tetrazolium bromide (MTT) was employed to demonstrate nicotinamide-adenine dinucleotide and nicotinamide-adenine dinucleotide phosphate diaphorases (NAD-D and NADP-D), succinic dehydrogenase (SDH), $\alpha$-glycerophosphate dehydrogenase ( $\alpha$-GPDH) using menaphthone as an intermediate hydrogen carrier, lactate dehydrogenase (LDH), malate dehydrogenase $(\mathrm{MDH})$, glucose-6-phosphate dehydrogenase (G6PDH), isocitrate dehydrogenase (ICDH), and $\beta$-hydroxybutyrate dehydrogenase ( $\beta$-OHBDH). Cytochrome oxidase (CytO) was demonstrated using $\alpha$-naphthol and 4-amino-N,N-dimethylnaphthylamine (ADN). Monoamine oxidase (MAO) was localized using 2,2',5,5'-tetra-p-nitrophenyl-3,3'-(3,3'-dimethoxy, 4,4'-biphenylene)-ditetrazolium chloride (Tetra-NitroBT). Ubiquinones (Ubq.) were identified by the method of Tranzer and Pearse (1963).

Sections for diaphorases and dehydrogenases were incubated 15 to 60 minutes at $37^{\circ} \mathrm{C}$. Those for CytO and MAO were incubated 60 and 90 minutes. Chloroform-extracted $2 \%$ methyl green was employed as a nuclear counterstain for all the preparations except those for CytO.

The possible influence of the gelatin on the oxidative enzymes was studied by comparing reactions in injected and uninjected lungs. The lungs from two of the rats, one euthanized by coal gas and the other by Nembutal, were not injected with gelatin. Small blocks of the collapsed lung were quenched with liquid oxygen and cut using the microtome cryostat. Only fragmentary sections of the collapsed lung could be cut, but the sections obtained were incubated in the same solutions and at the same time as gelatininfiltrated lung sections.

Sections of rat kidney and liver were incubated in the same solutions as the lung sections to determine whether or not the histochemical procedures were reacting properly. Histochemical controls consisted of sections of lung and other tissues which were 
incubated in media lacking substrate, co-enzyme or both.

The relative intensity of the overall reaction was estimated on sections incubated for 60 minutes and inspected without magnification and at $\times 25$. Overall reactions were graded as strong, moderate or weak. The intensity of the reaction in the various cells was estimated at $\times 400$ to $\times 1000$ and recorded as ++++ for very strong to \pm for very weak reactions.

The identification and nomenclature of the cells of the interalveolar septum has been extensively reviewed by Bertalanffy (1964), and his nomenclature was used in this study. Thus the cells were identified as alveolar cells, pulmonary epithelium or pulmonary endothelium. Free cells in the alveolar lumen were considered to be pulmonary macrophages and were not a primary object of this study as they are not part of the interalveolar septum.

\section{RESULTS}

The relative intensities of the reactions are presented in the Table. Overall reactions of control tissues were generally stronger than those of lung. Strong overall reactions in lung were observed for NAD-D, NADP-D, and Ubq. Overall reactions in lung for $\mathrm{MDH}, \mathrm{LDH}$, $\alpha-G P D H$ and CytO were judged to be moderate and those for $\beta$-OHBDH, ICDH, G6PDH, SDH, and MAO were estimated to be weak.

Typically the cells of the interalveolar septum had weaker reactions than those observed in other lung tissues, especially bronchial epithelium. Differences in the reaction of cells forming the blood-air pathway were observed only for CytO, and it was always difficult to differentiate the reactions of pulmonary epithelium from those of pulmonary endothelium. Generally the reactions were more intense in the alveolar cells than in the pulmonary epithelium or endothelium (Fig. 1). The

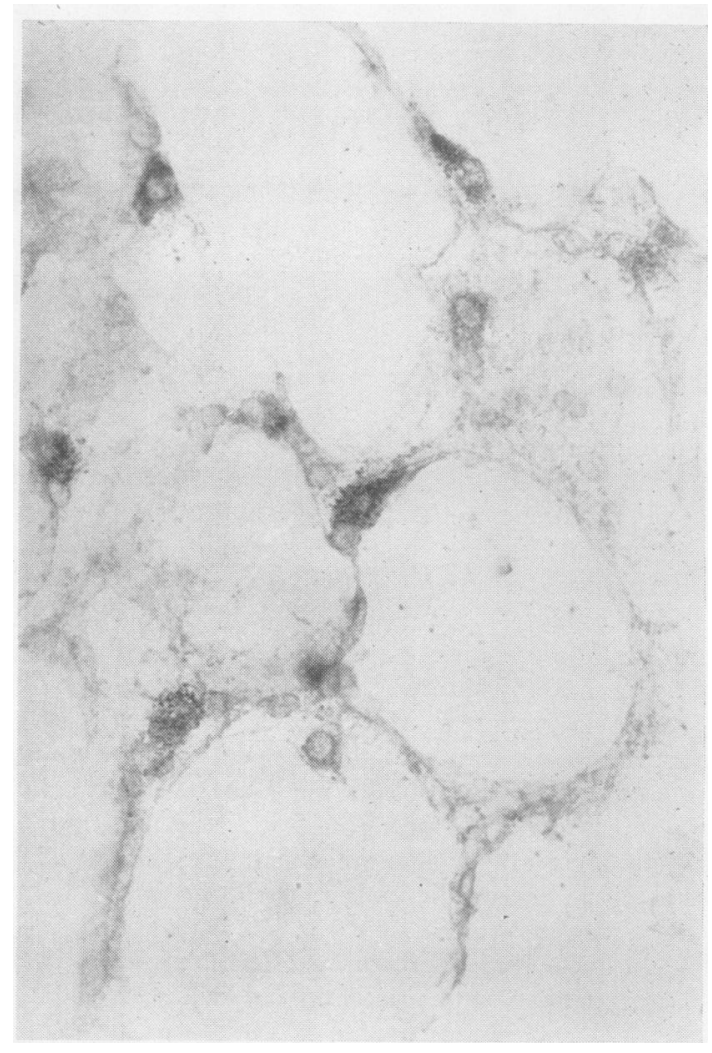

FIG. 1. NADP-D activity in rat interalveolar septa. The reaction is much stronger in the alveolar cells than in the pulmonary epithelium or pulmonary endothelium. Incubated $30 \min .8 \mu$. $\times 370$.

exceptions were the reactions for G6PDH, ICDH, and MAO, which appeared to have a more nearly uniform intensity in all three of these cell types (Fig. 2). The final reaction product in alveolar

T A B LE

HISTOCHEMICAL REACTIONS OF INTERALVEOLAR SEPTUM OF RAT FOR OXIDATIVE ENZYMES

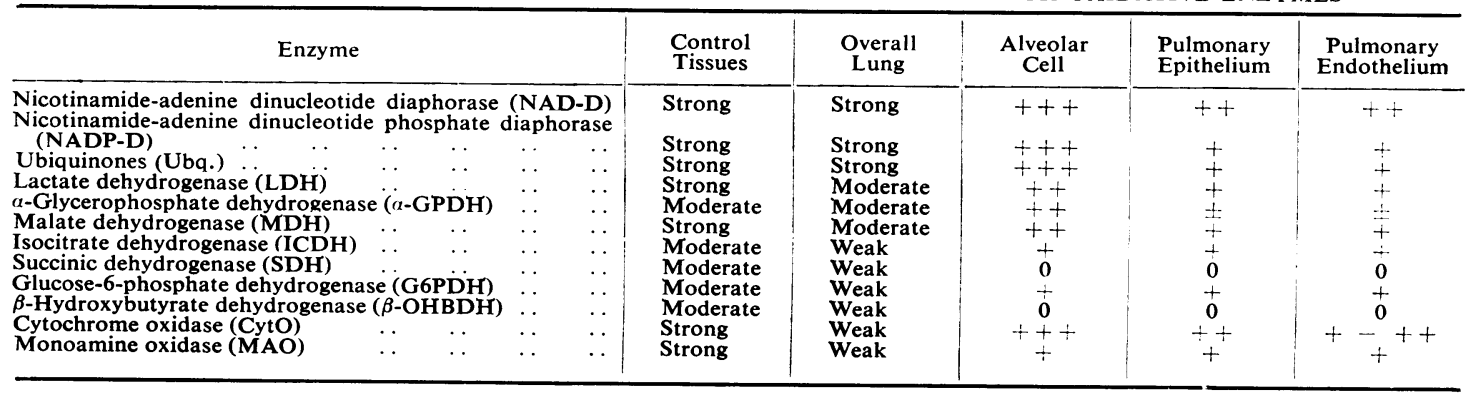




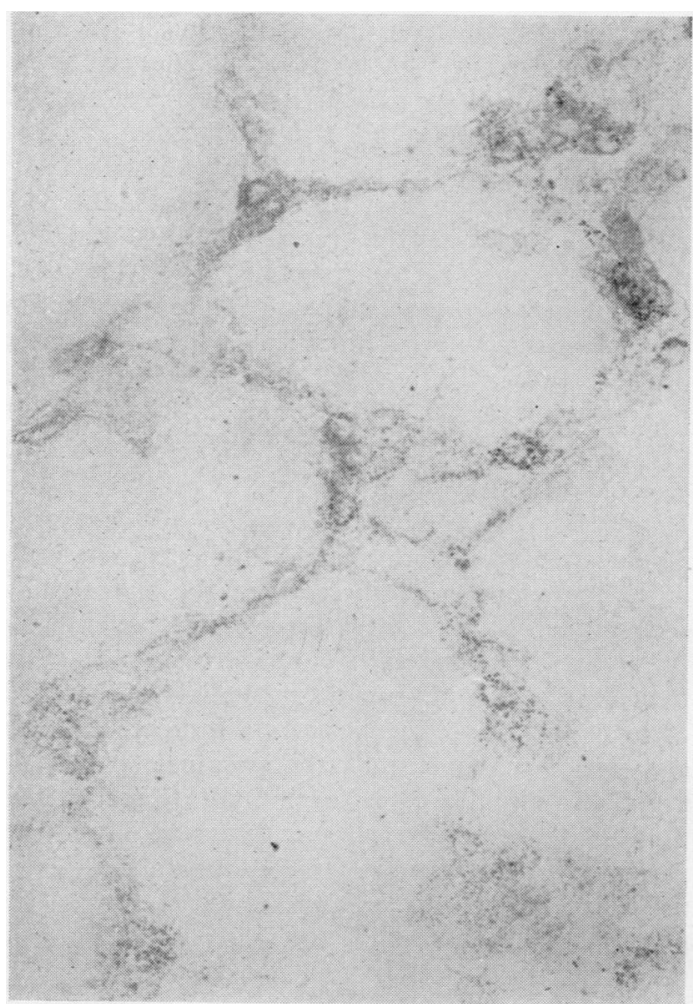

FIG. 2. ICDH activity in rat interalveolar septa. The reaction is nearly uniform in all cell types. The final reaction product appears in the form of coarser granules in the alveolar cells than in the epithelial or endothelial cells. Incubated 60 min. $20 \mu . \times 370$.

cells was usually in the form of coarser granules than that in the other cells. The reactions for $\beta$-OHBDH and SDH were essentially negative for the cells of the interalveolar septum, but reactions in other pulmonary tissues were estimated as + or ++ . Free cells in the alveolar lumen had reactions similar to those of the alveolar cells.

The reactions in control tissues were as expected on the basis of previous experience. Sections incubated in media lacking substrate or co-enzyme were negative.

No evidence of inhibition or translocation of enzymes due to the injection of gelatin were observed. Reactions in the fragments of uninjected lungs had similar intensities to those in injected lungs, but it was very difficult to determine cell types in the collapsed, uninjected lungs. No differences due to the method of euthanasia were detected.

\section{DISCUSSION}

These studies revealed that the alveolar cell has a greater potential for metabolic activity than the pulmonary epithelium or the pulmonary endothelium. The latter two cell types have nearly similar histochemical reactions. These observations correlate well with electron microscopic studies (Gillespie, Pangborn, Addison, and Tyler, 1964) which revealed more numerous mitochondria in the alveolar cells than in the pulmonary epithelium or endothelium. The alveolar cell may require this greater metabolic potential to produce the osmiophilic inclusions typical of this cell. These osmiophilic inclusions are thought by Klaus et al. (1962) to be the source of the pulmonary surfactant and our work reported here clearly supports their conclusions. The similarity between the pulmonary epithelium and the endothelium is supported by the observed similarity of their cytoplasm in the electron microscopic studies of De Groodt, Lagasse, and Sebruyns (1958).

Although exact correlations between enzymes demonstrated by histochemical and biochemical methods are not available for all the enzyme systems studied, speculation concerning the possible functional significance of these observations may contribute to a better understanding of structure-function relationships in the lung. Strong reactions for NAD-D and NADP-D often accompany high activities of NAD and NADP linked dehydrogenases. NADP linked dehydrogenases are frequently associated with biosynthetic systems. The strong reactions for NADP-D in alveolar cells are specially significant in view of the possible role of these cells in the production of pulmonary surfactant. The precise role of ubiquinones, or coenzymes $Q$, in the electron transport system is not known, but Green (1964) has suggested that they are important in the transfer of electrons across lipid layers from his complex II (or complex I) to complex III in mitochondria. CytO is the terminal oxidase of the cytochrome chain and transfers electrons to molecular oxygen. The concentration of this enzyme is directly correlated with oxygen requirement. MAO functions in the destruction or detoxication of amines including adrenalin and serotonin.

Reactions for enzymes of the glycolytic scheme, LDH and $\alpha$-GPDH, were moderately strong in alveolar cells, indicating that this metabolic pathway is potentially functional in these cells. Though the reaction for $\alpha-G P D H$ was very weak in 
pulmonary epithelium and endothelium, it is thought that the glycolytic scheme is functional in these cells. The presence of G6PDH in all the cells of the interalveolar septa indicates that the pentose cycle is potentially functional. This cycle could provide pentoses for nucleotide derivatives necessary for phospholipid synthesis. The absence of demonstrable $\beta$-OHBDH may indicate that fatty acid metabolism is much less important to the cells than the glycolytic scheme or the pentose cycle.

The results for the three histochemically demonstrable dehydrogenases of the tricarboxylic acid (TCA) cycle, MDH, ICDH, and SDH, were puzzling, and these data are difficult to interpret. Our own observations agree only in part with those of Sorokin et al. (1959), Sorokin (1961), and Pliess and Suhr (1959). In all these studies SDH was present and had the strongest reaction in vascular musculature. The studies of Sorokin et al. (1959) and Sorokin (1961) concerned in vivo and in vitro development of lungs. They did not specifically state the SDH activity in the cells of the interalveolar septum of their post-natal rats, but they did report a decrease in SDH activity of the stroma with increasing age. Their earlier studies were conducted before the development of MTT or Nitro-BT, and their later studies

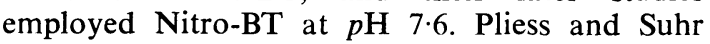
(1959) reported the presence of SDH in alveolar cells. However, they used very long (two to three hours) incubation times with a very easily reduced tetrazolium salt, Nitro-BT. The possibility of false localizations under the condition of these earlier studies has been discussed (Pearse, 1960) and must be considered. Using the MTT-cobalt chelation method we were able to demonstrate very weak reactions for SDH in the exchange area of avian lungs (Tyler and Pearse, 1964a), but found no activity in the interalveolar septa of horse lungs (Tyler and Pearse, 1964b). In our opinion, additional studies using other methods, probably those of Lowry, Roberts, Wu, Hixon, and Crawford (1954), are required to determine whether or not the TCA cycle is functional in the cells of the interalveolar septa.

The method used in this study to prepare cryostat sections of lung appears to be very satisfactory. The gelatin did not appear to cause inhibition or translocation of enzymes. These observations are in accord with those of Fishman, Ladue, and Borger (1961), who used gelatin in studies of free cell enzymorphology, and with our previous studies of the chicken lung (Tyler and Pearse, 1964a). Histochemical methods employing cryostat sections have many obvious applications to lungs from a variety of experimental procedures, and we anticipate that these methods will be very useful in the evaluation of early pathological changes in pulmonary tissues.

The observation that the method of euthanasia did not influence the results of histochemical procedures was not unexpected. However, it was thought to be an important observation when one of the agents entered directly through the tissues under study. Prolonged exposure to smaller quantities of either of the chemical agents might produce entirely different results.

\section{SUMMARY}

The distribution and relative intensity of several oxidative enzymes of the cells of the interalveolar septum have been investigated histochemically. Alveolar cells generally had stronger reactions than had the pulmonary epithelial cells or the endothelial cells. The reactions indicate that the glycolytic scheme and the pentose cycle are more active pathways of carbohydrate metabolism than is the tricarboxylic acid cycle. The histochemical observations were correlated with previous electron microscopic studies. The possible significance of the histochemical reactions of the alveolar cell to the synthesis of pulmonary surfactant is discussed.

The authors are grateful to Mr. J. D. Bancroft for technical assistance with many of the procedures. and to Mr. W. Brackenbury for the photomicrographs.

\section{REFERENCES}

Bertalanffy, F. D. (1964). Respiratory tissue : structure, histophysiology, cytodynamics. Part I. Review and basic cytomorphology, In Int. Rev. Cytol., 16, 233.

De Groodt, M., Lagasse, A., and Sebruyns, M. (1958). Fine structure of the alveolar wall of the lung. Nature (Lond.), 181, 1066.

Fishman, W. H., Ladue, K. T., and Borger, P. R. F. (1961). Enzymorphology : A study of free cells. J. Histochem. Cytochem., 9, 424. Gillespie, J., Pangborn, J., Addison, R., and Tyler, W. S. (1964). Ultrastructure of the pulmonary blood-air barrier of the horse. Anat. Rec., 148, 285.

Green, D. E. (1964). The mitochondrion. Scientific America, 2io, No. 1, p. 63.

Klaus, M. H. Reiss, O. K., Tooley, W. H., Piel, C., and Clements J. A. (1962). Alveolar epithelial cell mitochondria as source of the surface-active lung lining. Science, 137, 750 .

Lowry, O. H., Roberts, N. R., Wu, M. L., Hixon, W. S., and Crawford, E. J. (1954). The quantitative histochemistry of brain. Il. Enzyme measurement. J. biol. Chem., 207, 19.

Pearse, A. G. E. (1960). Histochemistry, Theoretical and Applied, 2nd ed. Churchill, London.

Pliess, G., and Suhr, A. (1959). Histochemische Befunde an der Rattenlunge nach Cortisonmedikation. Beitr. Path. Anat., 121, 406.

Sorokin, S. (1961). A study of development in organ cultures of mammalian lungs. Develop. Biol., 3, 60.

Padykula, H. A., and Herman, E. (1959). Comparative histochemical patterns in developing mammalian lungs. Ibid., 1 , 125.

Tranzer, J.-P., and Pearse, A. G. E. (1963). Cytochemical demonstration of ubiquinones in animal tissues. Nature (Lond.), 199, 1063.

Tyler, W. S., and Pearse, A. G. E. (1964a). Functional and analytica histochemistry of the chicken lung lobule with particular reference to surfactant. Amer. J. Anat. In press. 